\title{
Qualidade de vida e consumo de alcoólicos em hepatopatas do sexo masculino
}

\author{
Quality of life and alcohol consumption in male patients with liver diseases
}

\author{
Maria Evangelista Martins', Luiz Cláudio Ribeiro², Rafael Alves Baracho³, \\ Thales Januzzi Feital ${ }^{4}$, Mário Sérgio Ribeiro ${ }^{5}$
}

\author{
1 Psicóloga, mestre em Saúde, pesquisadora do Laboratório de Pesquisa em Personalidade, Álcool e Drogas da Universidade Federal de Juiz de Fora (LAPPDA-UFJF). \\ 2 Professor adjunto do Departamento de Estatística da UFJF, doutor em Demografia. \\ 3 Psicólogo, bolsista do LAPPDA. \\ ${ }^{4}$ Graduando em estatística, bolsista do LAPPDA. \\ ${ }_{5}$ Professor-associado do Departamento de Clínica Médica da UFJF, doutor em Filosofia, coordenador do LAPPDA-UFJF.
}

Recebido: 23/2/2011 - Aceito: 26/9/2011

\begin{abstract}
Resumo
Contexto: $\mathrm{O}$ uso disfuncional de álcool é um problema com amplas consequências. Além disso, os efeitos do seu uso sobre a qualidade de vida não foram ainda muito estudados. Objetivo: Avaliar a qualidade de vida de hepatopatas e verificar suas possíveis associações com o consumo de alcoólicos e variáveis sociodemográficas. Método: Estudo transversal realizado com pacientes atendidos em ambulatório de hepatologia, entre abril e dezembro de 2009, avaliando homens entre 20 e 59 anos e escolaridade superior à 5 a série do ensino fundamental. Instrumentos: CAGE, AUDIT, WHOQOL-Bref e questionário sociodemográfico. Resultados: Pacientes CAGE positivos tiveram médias mais baixas no domínio físico do WHOQOL ( $\mathrm{p}=0,027$ ). Aqueles que nunca usaram alcoólicos tiveram médias mais altas no domínio social comparados aos consumidores de médio e alto risco ou provável dependência no ano ( $\mathrm{p}$-valores, respectivamente, 0,023 e 0,036). Abstêmios e usuários de baixo risco registraram médias mais elevadas no domínio social comparados aos usuários de médio risco no último ano (p = 0,031). Conclusão: Os mais baixos níveis de qualidade de vida nos domínios físico (CAGE) e social (AUDIT) registrados pelos pacientes que informaram consumo de alcoólicos de alto risco ou provável dependência são consistentes com achados da literatura.
\end{abstract}

Martins ME, et al. / Rev Psiq Clín. 2012;39(1):5-11

Palavras-chave: WHOQOL-Bref, CAGE, AUDIT, uso de alcoólicos, qualidade de vida.

\begin{abstract}
Background: Dysfunctional use of alcohol is a problem with broad consequences. Moreover, there exists little evidence about the impact of dysfunctional use on quality of life. Objective: To evaluate the quality of life of patients with liver diseases and to verify its possible associations with alcohol consumption and sociodemographic variables. Method: Transverse study with masculine patients between 20 and 59 years of age and with at least 5 years of primary school completed, assessed between April and December 2009 in a hepatology service. Instruments: CAGE, AUDIT, WHOQOL-Bref and sociodemographic questionnaire. Results: CAGE positive patients had a lower average in the physical domain of WHOQOL ( $\mathrm{p}=0.027)$. Those who never used alcohol had higher scores in the social domain compared to patients with medium and high risk consumption or probable dependence in the past year $(\mathrm{p}=0.023$ and $\mathrm{p}=0.036$ respectively). Abstainers and low-risk users recorded the highest average in the social domain compared to medium risk users in the past year ( $\mathrm{p}=0.031$ ). Discussion: The lowest levels of quality of life in the physical (CAGE) and social (AUDIT) domains reported by patients with alcohol consumption of high risk or probable dependence are consistent with findings in the literature.
\end{abstract}

Martins ME, et al. / Rev Psiq Clín. 2012;39(1):5-11

Keywords: WHOQOL-Bref, CAGE, AUDIT, alcoholics use, quality of life.

\section{Introdução}

O uso disfuncional de alcoólicos é um grande problema para a saúde pública, constituindo-se em causa estimada de aproximadamente $4,5 \%$ das incapacidades, $3,8 \%$ (ou 2,5 milhões) do total de mortes no mundo em 2004․ É o maior fator de risco evitável de diversas patologias, entre essas a doença hepática alcoólica cujo espectro varia da esteatose a hepatite e cirrose ${ }^{1}$. Numa avaliação por escala de 1 a 5 pontos elaborada pela Organização Mundial de Saúde (OMS) para classificação dos prejuízos à saúde atribuídos ao uso disfuncional de álcool, o Brasil obteve o nível 3 no ano de 2005. Nesse mesmo ano, a taxa de mortalidade por cirrose hepática alcoólica na população brasileira acima de 15 anos de idade foi de 24,4 por 100.000 para habitantes do sexo masculino e 4,7 para a população feminina ${ }^{2}$.

Revisão de estudos realizados sobre os efeitos do beber pesado e beber pesado episódico no Brasil encontrou que há um consenso sobre as consequências negativas desse padrão de uso para a saúde e nas relações sociais 3 .

Apesar de o uso disfuncional de álcool ser um problema com amplas consequências, os efeitos do seu uso sobre a qualidade de vida não foram ainda muito estudados ${ }^{4}$. Todavia, a avaliação da qualidade de vida tem-se tornado um relevante critério na mensuração dos resultados terapêuticos em diferentes patologias crônicas: além de possibilitar a verificação da efetividade dos tratamentos em diferentes domínios, permite o direcionamento de estratégias terapêuticas para focos objetivos e mais específicos ${ }^{5}$.

Estudos de avaliação da qualidade de vida de pacientes hepatopatas utilizando o instrumento SF-36 têm evidenciado que sua qualidade de vida relacionada à saúde se mostra diminuída em relação a controles saudáveis ${ }^{6-8}$. Comparações entre grupos com base na gravidade da doença hepática indicaram piores níveis de qualidade de vida em pacientes com cirrose ${ }^{7,9}$, e pesquisas de seguimento que 
avaliaram pacientes submetidos a transplante hepático evidenciaram posterior incremento na qualidade de vida10,11. Poucos trabalhos avaliam o uso atual de alcoólicos em hepatopatas $7,11,12$ e poucos estudos avaliaram a qualidade de vida desses pacientes utilizando o instrumento WHOQOL-Bref 13,14 .

O objetivo deste estudo foi avaliar a qualidade de vida de pacientes portadores de hepatopatias e verificar sua possível modulação pelo consumo de alcoólicos.

\section{Métodos}

Estudo transversal realizado com pacientes em processo de avaliação diagnóstica ou tratamento no ambulatório de hepatologia do Serviço de Gastroenterologia do Centro de Atenção à Saúde da Universidade Federal de Juiz de Fora (CAS-UFJF). O ambulatório de hepatologia do CAS-UFJF é referência regional - Zona da Mata, sul de Minas e Campo das Vertentes - para atendimento a indivíduos que apresentam problemas hepáticos de diversas etiologias, alcoólicas e não alcoólicas.

Os critérios de elegibilidade para inclusão na pesquisa foram: sexo masculino, idade entre 20 e 59 anos e nível de escolaridade mínimo de $5^{\text {a }}$ série do ensino fundamental. Além do fato de o consumo de alcoólicos ser mais prevalente em homens ${ }^{15-17}$, a faixa etária escolhida é representativa do período de vida no qual os efeitos do uso disfuncional de alcoólicos é mais evidente ${ }^{18}$. Como critérios de não elegibilidade foram considerados: apresentar encefalopatia hepática, não ter condições de responder aos instrumentos de pesquisa (limitações cognitivas evidentes, incluindo intoxicação alcoólica) e recusa em assinar o Termo de Consentimento Livre e Esclarecido. Este estudo foi devidamente aprovado pelo Comitê de Ética em Pesquisa do Hospital Universitário (HU-UFJF).

Os instrumentos utilizados foram:

1. Questionário estruturado e autoaplicável relativo aos aspectos sociodemográficos.

2. Questionário CAGE de rastreamento de possível síndrome de dependência a alcoólicos na vida. Desenvolvido em 1968 por Ewing e Rouse, em estudo clínico realizado em um hospital geral nos Estados Unidos, com um grupo de 130 pacientes clínicos e cirúrgicos submetidos a uma extensa entrevista na qual responderam a perguntas elaboradas para a detecção do alcoolismo. Depois de se avaliar o menor número possível de questões suficientes para separar alcoolistas de não alcoolistas, concluiu-se pelo número definitivo de 4 (quatro) questões colocadas em uma ordem para possibilitar o recurso mnemônico (CAGE, gaiola, prisão) que nomeou o questionário ${ }^{19,20}$. Em estudo subsequente, concluiu-se que duas ou mais respostas afirmativas indicariam a possível dependência, com desempenho de $81 \%$ de sensibilidade e $89 \%$ de especificidade ${ }^{19-21}$. O instrumento foi padronizado e validado no Brasil, com sensibilidade e especificidade semelhante à original em língua inglesa ${ }^{22}$.

Para efeitos deste estudo, os sujeitos foram agrupados em "nunca usaram alcoólicos na vida", "CAGE-negativos" e "CAGE-positivos" (respostas afirmativas a duas ou mais perguntas).

3. Alcohol Use Disorders Identification Test (AUDIT), instrumento desenvolvido pela OMS como um método para investigação de uso disfuncional de alcoólicos no último ano (incluindo uso de risco), traduzido e validado no Brasil $^{23}$. Os domínios conceituais do AUDIT são: Consumo de álcool; Comportamento (dependência); Reações psicológicas adversas; Problemas relacionados ao álcool. As respostas às 10 questões do AUDIT recebem um valor entre 0 e 4, com um máximo de 40 pontos $^{24}$. A pontuação dos sujeitos no AUDIT foi categorizada em: "nunca usaram alcoólicos na vida"; "abstêmio recente ou bebedor de baixo risco" (de 0 a 7 pontos); "bebedores de médio risco (de 8 a 15 pontos); "bebedores de alto risco ou com provável diagnóstico" de transtorno mental relacionado ao uso de alcoólicos (16 ou mais pontos).
4. O instrumento WHOQOL-Bref, de avaliação da qualidade de vida, que é uma versão curta do WHOQOL-100 e contém 26 questões divididas em quatro domínios: físico, psicológico, relações sociais e meio ambiente, além de duas questões que se referem ao domínio geral - qualidade de vida global e percepções de saúde geral ${ }^{5,25}$. A versão abreviada em português do WHOQOL-Bref mostrou que o instrumento apresenta características satisfatórias de consistência interna, validade discriminante, validade de critério, validade concorrente e fidedignidade teste-reteste ${ }^{26}$. Os resultados relativos aos domínios do WHOQOL-Bref podem ser apresentados por meio de escores transformados em escalas de 4 a 20 ou de 0 a 100 pontos $^{25}$.

A coleta dos dados foi realizada por dois pesquisadores previamente treinados que compareciam ao ambulatório de Hepatologia nos dias e horários dos atendimentos médicos. Por meio de consultas prévias à agenda de marcação dos atendimentos médicos e aos prontuários dos pacientes, os pesquisadores elaboravam uma lista nominal daqueles agendados para cada dia de atendimento. Esses pacientes eram abordados na sala de espera da instituição e passavam por uma curta triagem voltada aos critérios de inclusão, após a qual os pacientes eram convidados a participar da pesquisa. Para os que concordavam, eram explicados os objetivos, esclarecidos os procedimentos éticos e legais e obtida a assinatura do Termo de Consentimento Livre e Esclarecido.

Todos os cuidados foram tomados para que os participantes não compartilhassem ou discutissem os conteúdos dos instrumentos com acompanhantes ou pessoas alheias à pesquisa. Para tanto, havia sempre um pesquisador presente durante toda a aplicação. A maioria dos questionários foi respondida em uma única etapa, antes da consulta agendada. Quando o sujeito era chamado à consulta antes de concluir o preenchimento do questionário, o pesquisador aguardava seu retorno à sala de espera para a conclusão dele. Em 27 situações o questionário precisou ser completado no retorno do paciente para nova consulta; nessa eventualidade, teve-se o cuidado de garantir que o WHOQOL-BREF fosse respondido em uma única ocasião, como preconizado 5 .

Foram abordados 354 pacientes do sexo masculino que compareceram às consultas agendadas no período de abril a dezembro de 2009, e o processo só foi interrompido quando se observou que praticamente todos pacientes que compareciam às novas consultas já haviam sido abordados. Desses 354 sujeitos, 14 se recusaram a participar, 165 não se enquadravam nos critérios de elegibilidade para inclusão na pesquisa; 69 não apresentavam condições de escolaridade mínima; 80 estavam fora da faixa etária estabelecida e 7 tanto estavam fora da faixa etária quanto também não apresentavam condições de escolaridade mínima. Resposta ao questionário de forma incompleta ou incoerente, tal como relatar consumo de álcool no último ano e em seguida responder que nunca fez uso de alcoólicos implicou a não inclusão na análise de 48 sujeitos. Assim sendo, 127 pacientes foram incluídos no estudo (Figura 1): todos haviam respondido a quatro perguntas do CAGE e a oito ou mais perguntas do AUDIT e satisfizeram os critérios estabelecidos pelo grupo WHOQOL ${ }^{5}$ para considerar o questionário válido para análise. De acordo com as recomendações do grupo WHOQOL, todos os 127 casos analisados responderam a mais de $80 \%$ dos itens do questionário; quando alguma pergunta do domínio social e mais de dois itens dos outros três domínios não são respondidos, o escore do domínio não deve ser calculado: tal recomendação levou a que indivíduos sem pontuação em algum domínio não fossem incluídos nas análises relativas a esse domínio específico ${ }^{5}$

O banco de dados foi construído utilizando-se o programa SPSS for Windows 14.0 (número de série 9656438), por meio do qual os dados foram submetidos às análises.

A qualidade de vida, variável dependente deste estudo, foi avaliada pelo critério do "escore transformado", em escalas de 0 a 100 para cada um dos quatro domínios 5 .

Para efeito das análises bivariadas, foram utilizados apenas os casos válidos, e as variáveis independentes foram agrupadas da seguinte 


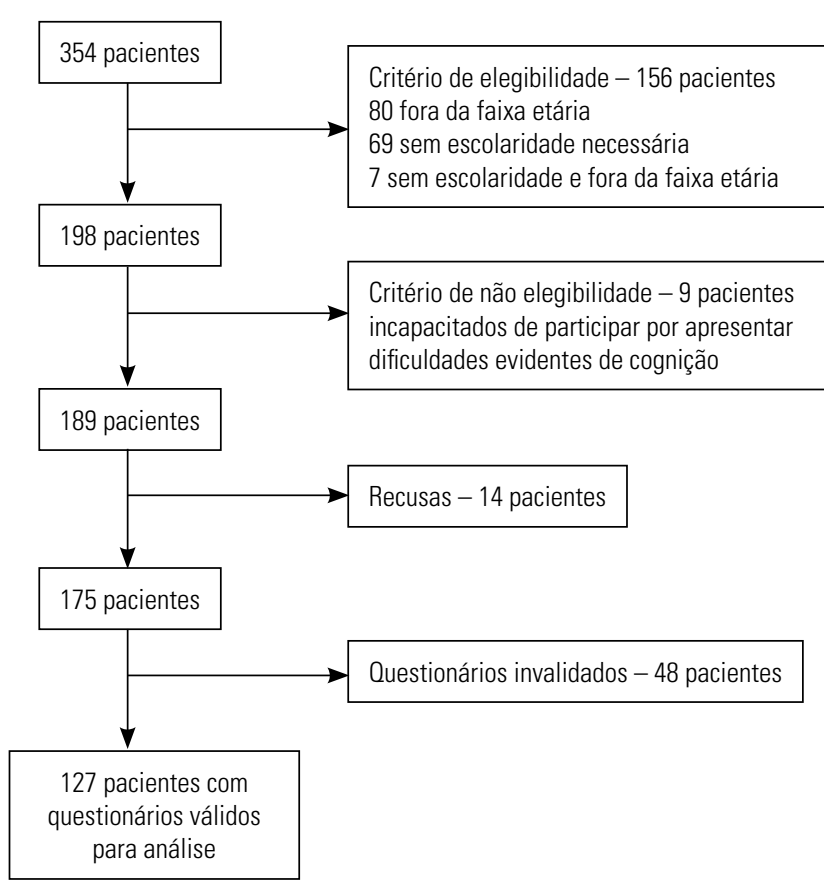

Figura 1. Fluxograma dos pacientes abordados para participação na pesquisa.

forma: a idade foi categorizada em " 20 a 30", “31 a 40 ", "41 a 50 ” e "51 a 60" anos; o estado civil foi agrupado em "solteiros", "casados ou amigados" e "outros" (viúvos, divorciados e separados); a escolaridade, em "Ensino fundamental completo", "Ensino médio incompleto ou completo" e "Ensino superior incompleto ou acima"; a ocupação, em "não trabalha", "trabalha" e "estudante (empregado ou não)"; a remuneração, em "menor ou igual a 1 salário-mínimo", "mais de 1 até 3 " e "acima de 3 salários-mínimos"; e a religião foi categorizada em "não possui", "católica", "evangélicos/protestantes" e "outras"; na frequência aos cultos religiosos, as categorias foram "Não possui religião"; "Não participa de forma regular" e "Participa regularmente".

Para as análises bivariadas, os resultados do CAGE e do AUDIT foram combinados e analisados de acordo com seis subgrupos resultantes: Grupo I ("nunca usaram alcoólicos"), Grupo II ("CAGE negativo e AUDIT de 0 a 7 pontos", constituindo-se no grupo de sujeitos não identificados como prováveis dependentes na vida e abstêmios ou com consumo de baixo risco de alcoólicos no último ano); Grupo III ("CAGE negativo e AUDIT de 8 a 15 pontos", ou seja, não identificados como prováveis dependentes na vida e com consumo de médio risco no último ano); Grupo IV ("CAGE positivo e AUDIT de 0 a 7 pontos”, sujeitos com provável síndrome de dependência alcoólica na vida e abstêmios ou com consumo de baixo risco no último ano); Grupo V ("CAGE positivo e AUDIT de 8 a 15 pontos", indivíduos com provável síndrome de dependência alcoólica na vida e consumo de médio risco no último ano) e Grupo VI ("CAGE positivo e AUDIT de 16 ou mais pontos", com provável síndrome de dependência alcoólica na vida e consumo de alto risco ou provável dependência no último ano).

Por meio de teste de normalidade, foi observado que as categorias presentes nas variáveis independentes não apresentavam distribuição normal da pontuação dos indivíduos nos domínios do WHOQOL-Bref. As possíveis diferenças entre os grupos estudados foram verificadas por meio do teste não paramétrico de Kruskal-Wallis e para as comparações múltiplas ${ }^{27}$ foi utilizado o teste de Conover-Inman:

$T_{\mathrm{r}}=\frac{\left|\bar{R}_{i}-\bar{R}_{j}\right|}{\sqrt{S_{\mathrm{r}}^{2}\left(\frac{N-1-H}{N-k}\right)\left(\frac{1}{n_{i}}+\frac{1}{n_{j}}\right)}}$, com uma distribuição t, onde as médias ranqueadas, $\mathrm{N}$ é o tamanho total da amostra (população no caso do presente estudo), $n_{i}$ e $n_{j}$ referem-se ao tamanho de cada grupo que está sendo comparado, k é o número de grupos e H é a estatística de teste do Kruskal-Wallis. Para efeitos das análises aqui realizadas, os resultados foram considerados significantes quando os $\mathrm{p}$-valores foram $\leq 0,05$ e marginalmente significantes quando $0,1<p>0,05$.

\section{Resultados}

A média de idade dos sujeitos estudados foi de 43,02 (+/-9,73) anos; em sua maioria eram casados $(56,7 \%)$, com escolaridade de até o Ensino médio completo $(87,4 \%)$ e $81,7 \%$ com renda mensal de até 3 salários-mínimos; $38,6 \%$ trabalhavam no momento da avaliação. Professar a religião católica foi referido por $62,2 \%$ dos sujeitos; $62,2 \%$ frequentavam o culto de sua religião e $44,1 \%$ o faziam de forma semanal.

As pontuações médias no WHOQOL-Bref, por domínio, foram: físico (60,96; DP 19,5), psicológico (68,71; DP 15,39), social $(67,54$; DP 18,33$)$ e ambiental $(57,02$; DP 12,77).

Dos 127 sujeitos estudados, $14,2 \%$ informaram nunca ter feito uso de alcoólicos e 44,9\% foram identificados como CAGE-positivos, isto é, rastreados como prováveis dependentes de alcoólicos na vida. Para o AUDIT, além dos 14,2\% que nunca consumiram alcoólicos, $54,3 \%$ apresentaram pontuação inferior a 8 pontos - indicativo de ausência de consumo no último ano ou uso em padrão considerado como de baixo risco - e $31,5 \%$ pontuaram em níveis indicativos de uso de médio a alto risco ou provável dependência no ano de alcoólicos.

Os resultados das análises bivariadas entre qualidade de vida avaliada pelos domínios do WHOQOL-Bref e as variáveis estudadas estão apresentados nas tabelas 1 a 3 .

\section{Características sociodemográficas e qualidade de vida}

Para as variáveis sociodemográficas, diferenças significantes quanto à qualidade de vida foram registradas apenas para as variáveis faixa etária e ocupação.

Os sujeitos na faixa etária de " 20 a 30 anos" $(\mathrm{n}=17)$ pontuaram de forma mais elevada em todos os domínios do WHOQOL-Bref, e as pontuações no domínio físico diminuíram progressivamente na medida em que aumentava a faixa etária. $\mathrm{O}$ teste de comparações múltiplas indicou que, no domínio físico, a diferença foi significante na comparação entre as faixas etárias de 20-30 com 41-50 anos $(\mathrm{p}=0,025)$ e 20-30 com 51-60 anos ( $\mathrm{p}=0,027)$. No domínio social, observou-se significância entre as faixas de 20-30 e 41-50 anos ( $\mathrm{p}=0,005)$.

Quanto à ocupação, os pacientes categorizados como "Estudante (empregado ou não)" atingiram as maiores médias em todos os domínios, e resultados estatisticamente significantes foram observados nos domínios físico e social (respectivamente $\mathrm{p}=0,01$ e $\mathrm{p}=0,046$ ). O teste de comparações múltiplas indicou que, no domínio físico, existem diferenças entre o grupo dos que informaram não trabalhar tanto com o grupo de estudantes quanto com o grupo dos que informaram trabalhar (p-valores de, respectivamente, 0,025 e 0,007). No domínio social, diferença estatisticamente significante foi observada entre o grupo que trabalha e o que não trabalha $(p=0,027)$.

\section{Consumo de alcoólicos e qualidade de vida}

As análises bivariadas entre qualidade de vida e CAGE revelaram que os sujeitos considerados como CAGE-negativos tiveram médias mais elevadas nos domínios físico, psicológico e ambiental, enquanto aqueles que afirmaram nunca ter usado alcoólicos tiveram mais altos escores no domínio social. Os CAGE-positivos registraram médias mais baixas em todos os domínios. Pelo teste de Kruskal-Wallis, apenas no domínio físico observou-se diferença marginalmente significante ( $\mathrm{p}=0,085)$; pelo teste de comparações múltiplas, identificou-se diferença plenamente significante entre os grupos Cage positivo e Cage negativo $(\mathrm{p}=0,027)$. 
Tabela 1. Variáveis sociodemográficas e pontuações médias (M) e desvio-padrão (DP) nos domínios do WHOQOL-Bref

\begin{tabular}{|c|c|c|c|c|c|c|c|c|}
\hline \multirow{3}{*}{\begin{tabular}{|l} 
\\
Variáveis \\
Faixa etária \\
\end{tabular}} & \multicolumn{8}{|c|}{ Domínios do WHO0OL-Bref em escala de 0-100 pontos } \\
\hline & \multicolumn{2}{|l|}{ Físico } & \multicolumn{2}{|c|}{ Psicológico } & \multicolumn{2}{|l|}{ Social } & \multicolumn{2}{|c|}{ Ambiental } \\
\hline & $\mathrm{M}(\mathrm{DP})$ & $\mathrm{N}$ & $\mathrm{M}(\mathrm{DP})$ & $\mathrm{N}$ & $\mathrm{M}(\mathrm{DP})$ & $\mathrm{N}$ & $\mathrm{M}(\mathrm{DP})$ & $\mathrm{N}$ \\
\hline $20-30$ & $70,37(15,30)^{1}$ & 17 & $73,52(14,12)$ & 17 & $77,45(17,12)^{2}$ & 17 & $59,55(12,13)$ & 17 \\
\hline $31-40$ & $64,63(17,08)$ & 31 & $67,20(15,16)$ & 31 & $68,33(15,22)$ & 30 & $54,03(9,85)$ & 31 \\
\hline $41-50$ & $57,61(19,16)^{1}$ & 45 & $68,61(15,65)$ & 45 & $63,25(18,79)^{2}$ & 44 & $57,88(13,18)$ & 44 \\
\hline $51-60$ & $57,25(21,15)$ & 33 & $67,80(16,07)$ & 33 & $67,42(19,58)$ & 33 & $57,38(14,57)$ & 33 \\
\hline $\mathrm{p}$-valor & \multicolumn{2}{|l|}{$0,068^{* *}$} & \multicolumn{2}{|l|}{0,566} & \multicolumn{2}{|l|}{$0,040^{*}$} & \multicolumn{2}{|l|}{0,315} \\
\hline \multicolumn{9}{|l|}{ Estado civil } \\
\hline Solteiro & $62,12(16,96)$ & 38 & $66,77(16,65)$ & 38 & $65,99(19,87)$ & 37 & $56,82(12,90)$ & 38 \\
\hline Casado/amigado & $61,16(20,10)$ & 71 & $70,18(14,80)$ & 71 & $69,28(17,80)$ & 70 & $56,99(13,08)$ & 71 \\
\hline Outros & $56,91(20,92)$ & 16 & $66,14(15,35)$ & 16 & $63,54(17,71)$ & 16 & $57,70(12,21)$ & 15 \\
\hline $\mathrm{p}$-valor & \multicolumn{2}{|l|}{0,806} & \multicolumn{2}{|l|}{0,528} & \multicolumn{2}{|l|}{0,435} & \multicolumn{2}{|l|}{0,821} \\
\hline \multicolumn{9}{|l|}{ Escolaridade } \\
\hline Até Ensino fundamental completo & $59,93(17,65)$ & 55 & $68,93(15,69)$ & 55 & $69,44(17,66)$ & 54 & $56,42(12,82)$ & 55 \\
\hline Ensino médio incompleto a completo & $61,16(21,44)$ & 16 & $68,25(15,84)$ & 16 & $64,96(19,78)$ & 16 & $56,01(12,09)$ & 16 \\
\hline Ensino superior incompleto acima & $63,83(16,38)$ & 16 & $69,53(13,58)$ & 16 & $69,79(15,17)$ & 16 & $62,50(13,92)$ & 16 \\
\hline $\mathrm{p}$-valor & \multicolumn{2}{|l|}{0,661} & \multicolumn{2}{|l|}{0,968} & \multicolumn{2}{|l|}{0,351} & \multicolumn{2}{|l|}{0,187} \\
\hline \multicolumn{9}{|l|}{ Ocupação } \\
\hline Estudante & $67,38(18,05)^{3}$ & 15 & $73,05(14,24)$ & 15 & $72,77(16,50)$ & 15 & $60,20(13,28)$ & 15 \\
\hline Trabalha & $65,25(18,10)^{4}$ & 48 & $69,47(14,25)$ & 49 & $71,35(16,50)^{5}$ & 48 & $56,70(13,28)$ & 48 \\
\hline Não trabalha & $54,82(19,85)^{3,4}$ & 54 & $66,98(14,79)$ & 53 & $62,89(19,44)^{5}$ & 53 & $56,36(12,90)$ & 53 \\
\hline $\mathrm{p}$-valor & \multicolumn{2}{|l|}{$0,010^{*}$} & \multicolumn{2}{|l|}{0,349} & \multicolumn{2}{|l|}{$0,046^{*}$} & \multicolumn{2}{|l|}{0,566} \\
\hline \multicolumn{9}{|l|}{ Remuneração } \\
\hline Ate 1 salario-mínimo & $58,81(20,41)$ & 49 & $69,01(15,27)$ & 48 & $67,37(19,95)$ & 47 & $56,44(13,93)$ & 49 \\
\hline Acima de 1 a 3 salários-mínimos & $62,79(18,41)$ & 48 & $68,28(16,37)$ & 49 & $67,68(18,44)$ & 49 & $54,85(11,13)$ & 47 \\
\hline Acima de 3 salários-mínimos & $60,87(18,97)$ & 22 & $67,99(15,23)$ & 22 & $66,66(15,13)$ & 21 & $61,07(13,38)$ & 22 \\
\hline $\mathrm{p}$-valor & \multicolumn{2}{|l|}{0,775} & \multicolumn{2}{|l|}{0,937} & \multicolumn{2}{|l|}{0,873} & \multicolumn{2}{|l|}{0,254} \\
\hline
\end{tabular}

p-valor $<0,05$.

*0,05<p-valor $<0,1$ (marginalmente significante).

1 p-valor do teste de comparação múltipla de Conover-Inman: 0,025.

2 p-valor do teste de comparação múltipla de Conover-Inman: 0,005.

${ }^{3}$ p-valor do teste de comparação múltipla de Conover-Inman: 0,025.

4 p-valor do teste de comparação múltipla de Conover-Inman: 0,007.

${ }^{5}$ p-valor do teste de comparação múltipla de Conover-Inman: 0,027.

Tabela 2. Variáveis religiosas e pontuações médias (M) e desvio-padrão (DP) nos domínios do WHOQOL-Bref

\begin{tabular}{|c|c|c|c|c|c|c|c|c|}
\hline \multirow{3}{*}{\begin{tabular}{|l} 
Variáveis \\
Religião \\
\end{tabular}} & \multicolumn{8}{|c|}{ Domínios do WHO0OL-Bref em escala de 0-100 pontos } \\
\hline & \multicolumn{2}{|l|}{ Físico } & \multicolumn{2}{|c|}{ Psicológico } & \multirow{2}{*}{$\begin{array}{c}\text { Social } \\
\mathrm{M}(\mathrm{DP}) \\
\end{array}$} & \multicolumn{3}{|c|}{ Ambiental } \\
\hline & $\mathrm{M}(\mathrm{DP})$ & $\mathrm{N}$ & $\mathrm{M}(\mathrm{DP})$ & $\mathrm{N}$ & & $\mathrm{N}$ & $\mathrm{M}(\mathrm{DP})$ & $\mathrm{N}$ \\
\hline Sem religião & $61,50(15,23)$ & 9 & $65,27(13,01)$ & 9 & $71,29(16,19)$ & 9 & $61,11(12,21)$ & 9 \\
\hline Católicos & $61,67(19,48)$ & 78 & $70,29(14,57)$ & 78 & $68,85(18,87)$ & 76 & $56,97(12,36)$ & 78 \\
\hline Protestantes/Evangélicos & $54,65(19,98)$ & 23 & $62,86(17,40)$ & 23 & $62,31(16,64)$ & 23 & $53,39(14,34)$ & 23 \\
\hline Outros & $64,79(18,25)$ & 14 & $69,79(17,15)$ & 14 & $65,47(20,11)$ & 14 & $61,16(12,36)$ & 14 \\
\hline p-valor & \multicolumn{2}{|l|}{0,338} & \multicolumn{2}{|l|}{0,232} & 0,499 & \multicolumn{3}{|c|}{0,257} \\
\hline \multicolumn{9}{|l|}{ Frequenta cultos religiosos } \\
\hline Não & $59,34(16,58)$ & 39 & $68,20(12,86)$ & 38 & $70,17(17,07)$ & 38 & $57,93(9,90)$ & 39 \\
\hline Sim & $62,25(20,44)$ & 79 & $70,09(16,06)$ & 79 & $67,74(18,99)$ & 77 & $57,54(13,84)$ & 77 \\
\hline $\mathrm{p}$-valor & \multicolumn{2}{|l|}{0,417} & \multicolumn{2}{|l|}{0,294} & 0,411 & \multicolumn{3}{|c|}{0,799} \\
\hline \multicolumn{9}{|l|}{ Frequência a cultos religiosos } \\
\hline Sem religião & $61,50(15,23)$ & 9 & $65,27(13,01)$ & 9 & $71,29(16,19)$ & 9 & $61,11(12,21)$ & 9 \\
\hline Não participa de forma regular & $60,88(17,05)$ & 41 & $67,70(15,00)$ & 40 & $65,17(20,13)$ & 39 & $55,03(10,52)$ & 41 \\
\hline Participa regularmente & $61,71(20,60)$ & 68 & $69,73(16,33)$ & 68 & $68,62(17,81)$ & 68 & $57,90(13,86)$ & 66 \\
\hline $\mathrm{p}$-valor & \multicolumn{2}{|l|}{0,966} & \multicolumn{2}{|l|}{0,458} & 0,649 & \multicolumn{3}{|c|}{0,317} \\
\hline
\end{tabular}

Em relação ao padrão de uso de alcoólicos no último ano, observou-se que os hepatopatas incluídos na categoria "bebedores de alto risco ou com provável dependência" apresentaram os menores escores em todos os domínios do WHOQOL-Bref. O teste de Kruskal-Wallis apontou para diferença significante apenas no domínio social $(\mathrm{p}=0,029)$, único domínio no qual os pacientes que relataram nunca terem usado alcoólicos tiveram o mais alto escore. O teste de comparações múltiplas revelou que essa diferença foi identificada tanto na comparação entre os sujeitos que informaram nunca terem usado alcoólicos e os consumidores de médio risco 
Tabela 3. Consumo de alcoólicos na vida e no último ano (classificações no CAGE e no AUDIT) e pontuações médias (M) e desvio-padrão (DP) nos domínios do WHOQOL-Bref

\begin{tabular}{|c|c|c|c|c|c|c|c|c|}
\hline \multirow{3}{*}{$\begin{array}{l}\text { Variáveis } \\
\text { Uso de álcool na vida (CAGE) }\end{array}$} & \multicolumn{8}{|c|}{ Domínios do WH000L-Bref em escala de 0-100 pontos } \\
\hline & \multicolumn{2}{|l|}{ Físico } & \multicolumn{2}{|c|}{ Psicológico } & \multicolumn{2}{|l|}{ Social } & \multicolumn{2}{|c|}{ Ambiental } \\
\hline & $\mathrm{M}(\mathrm{DP})$ & $\mathrm{N}$ & $\mathrm{M}(\mathrm{DP})$ & $\mathrm{N}$ & $\mathrm{M}(\mathrm{DP})$ & $\mathrm{N}$ & $\mathrm{M}(\mathrm{DP})$ & $\mathrm{N}$ \\
\hline Nunca usaram & $61,13(16,84)$ & 17 & $69,67(15,44)$ & 18 & $73,61(16,48)$ & 17 & $57,29(13,08)$ & 17 \\
\hline Negativos & $65,31(18,50)^{1}$ & 52 & $69,95(15,48)$ & 50 & $69,50(16,28)$ & 51 & $58,27(13,83)$ & 51 \\
\hline Positivos & $56,95(19,80)^{1}$ & 57 & $67,26(15,45)$ & 56 & $68,83(20,95)$ & 56 & $55,80(11,74)$ & 56 \\
\hline p-valor & \multicolumn{2}{|l|}{$0,085^{* *}$} & \multicolumn{2}{|l|}{0,663} & \multicolumn{2}{|l|}{0,127} & \multicolumn{2}{|l|}{0,530} \\
\hline \multicolumn{9}{|l|}{ Uso de álcool no último ano (AUDIT) } \\
\hline Nunca usaram & $61,13(16,84)$ & 17 & $69,67(15,44)$ & 18 & $73,61(16,48)^{3,4}$ & 18 & $57,29(13,08)$ & 18 \\
\hline $\begin{array}{l}\text { Abstêmios recentes ou consumo de baixo risco } \\
\text { (0-7 pontos ) }\end{array}$ & $61,69(19,97)$ & 69 & $70,22(15,47)$ & 69 & $70,27(16,49)^{2}$ & 67 & $58,11(12,93)$ & 67 \\
\hline Consumo de médio risco (8-15 pontos) & $62,32(17,82)$ & 31 & $67,50(14,53)$ & 30 & $60,55(21,43)^{2,3}$ & 30 & $56,75(12,68)$ & 31 \\
\hline $\begin{array}{l}\text { Consumo de alto risco ou provável dependência } \\
\text { (16 pontos ou mais) }\end{array}$ & $50,39(21,08)$ & 9 & $59,25(16,37)$ & 9 & $58,33(16,13)^{4}$ & 9 & $49,30(10,21)$ & 9 \\
\hline $\mathrm{p}$-valor & \multicolumn{2}{|l|}{0,448} & \multicolumn{2}{|l|}{0,225} & \multicolumn{2}{|l|}{$0,029^{*}$} & \multicolumn{2}{|l|}{0,273} \\
\hline \multicolumn{9}{|l|}{ Uso de alcoólicos na vida e no último ano } \\
\hline Grupo I: Nunca usaram & $61,13(16,84)$ & 17 & $69,67(15,44)$ & 18 & $73,61(16,48)$ & 18 & $57,29(10,21)$ & 18 \\
\hline Grupo II: CAGE negativo e AUDIT 0-7 pontos & $65,09(18,57)$ & 44 & $69,88(15,71)$ & 44 & $71,03(16,07)$ & 42 & $58,06(13,89)$ & 43 \\
\hline Grupo III: CAGE negativo e AUDIT 8-15 pontos & $65,51(19,27)$ & 8 & $70,31(15,17)$ & 8 & $61,45(16,02)$ & 8 & $59,37(14,36)$ & 8 \\
\hline Grupo IV: CAGE positivo e AUDIT 0-7 pontos & $55,71(21,30)$ & 25 & $70,83(15,35)$ & 25 & $69,00(17,43)$ & 25 & $58,20(11,27)$ & 25 \\
\hline Grupo V: CAGE positivo e AUDIT 8-15 pontos & $60,86(17,51)$ & 23 & $66,47(14,51)$ & 22 & $60,22(23,41)$ & 22 & $55,84(12,25)$ & 23 \\
\hline Grupo VI: CAGE positivo e AUDIT 16 pontos ou mais & $50,39(21,08)$ & 9 & $59,25(16,37)$ & 9 & $58,33(16,13)$ & 9 & $49,30(10,21)$ & 9 \\
\hline $\mathrm{p}$-valor & \multicolumn{2}{|l|}{0,249} & \multicolumn{2}{|l|}{0,468} & \multicolumn{2}{|l|}{0,101} & \multicolumn{2}{|l|}{0,477} \\
\hline
\end{tabular}

p-valor $<0,05$

**0,05<p-valor $<0,1$ (marginalmente significante).

i $\mathrm{p}$-valor do teste de comparação múltipla de Conover-Inman: 0,027.

2 -valor do teste de comparação múltipla de Conover-Inman: 0,031

${ }^{3} \mathrm{p}$-valor do teste de comparação múltipla de Conover-Inman: 0,023

${ }_{4}^{4}$ p-valor do teste de comparação múltipla de Conover-Inman: 0,036.

no último ano $(\mathrm{p}=0,023)$ quanto com consumidores de alto risco no último ano $(p=0,036)$. Diferença significante também foi registrada na comparação entre o grupo de abstêmios e usuários de baixo risco com os usuários de médio risco no último ano $(\mathrm{p}=0,031)$.

Vale ressaltar que os hepatopatas identificados como "CAGE negativos" ou no grupo de "abstêmios ou bebedores de baixo risco" no último ano obtiveram pontuações mais elevadas que todos os demais subgrupos - inclusive os que nunca usaram alcoólicos na vida - nos domínios psicológico e ambiental.

A variável resultante da combinação dos resultados do CAGE e do AUDIT não resultou em diferenças significantes quanto aos níveis de qualidade de vida dos sujeitos. Todavia, os resultados sugerem que a percepção da qualidade de vida por parte dos hepatopatas aqui estudados se correlacione a modificações no padrão de consumo de alcoólicos. Sujeitos identificados como CAGE-positivos apresentaram os mais baixos níveis de qualidade de vida no domínio físico; por sua vez, os resultados da variável combinada indicam que os sujeitos com consumo identificado como de médio risco pelo AUDIT se avaliaram com melhor nível no domínio físico quando comparados aos que, no último ano, se mantiveram abstinentes ou com consumo de baixo risco; e a pontuação mais baixa foi encontrada no subgrupo de CAGE-positivos identificados pelo AUDIT com consumo de alto risco ou com provável dependência no último ano. A manutenção de padrão de consumo de alcoólicos, nos últimos 12 meses, compatível com diagnóstico de síndrome de dependência também se traduziu pela pior média no domínio social; todavia, inversamente ao observado para o domínio físico, sujeitos CAGE-positivos que foram identificados pelo AUDIT como tendo padrão de médio risco no último ano referiram pior nível de qualidade de vida no domínio social. Para os sujeitos considerados como CAGE-negativos, o padrão de consumo de médio risco no último ano resultou em discreta elevação do nível de qualidade de vida no domínio físico, mas em importante queda no domínio social.

\section{Discussão}

Os sujeitos aqui estudados obtiveram valores médios semelhantes aos do estudo de normatização brasileira do WHOQOL-Bref ${ }^{28}$ nos domínios físico, psicológico e ambiental. Os resultados também se aproximam dos encontrados por Rocha e Fleck ${ }^{29}$ - que avaliaram a qualidade de vida de pacientes com doenças crônicas na cidade de Porto Alegre - nos domínios social e ambiental.

\section{Qualidade de vida e variáveis sociodemográficas}

Os resultados deste estudo indicaram associações entre idade e qualidade de vida apenas para os domínios físico e social. O domínio físico avalia facetas como dor e desconforto, energia e fadiga, sono e repouso, mobilidade, atividade de vida cotidiana, dependência de medicação ou de tratamentos e capacidade de trabalho: assim sendo, esta associação seria esperável e já foi encontrada em estudos que avaliaram a qualidade de vida em hepatopatas ${ }^{14,30}$. No campo das relações sociais, os resultados encontrados também não diferiram de estudos anteriores, que encontraram escores mais elevados entre os mais jovens ${ }^{8,31}$. Os achados relativos à ocupação - pacientes que não trabalhavam tiveram níveis mais baixos de qualidade de vida nos domínios físico e social que aqueles que estudavam e/ou estavam empregados - podem refletir um aspecto específico do domínio físico que é a avaliação da capacidade para o trabalho e são consistentes com o resultado do estudo de Ohaeri et al. ${ }^{32}$.

\section{Consumo de alcoólicos e qualidade de vida}

O resultado encontrado no domínio físico do WHOQOL-Bref para o uso de alcoólicos ao longo da vida não só é esperável - se se considerar os efeitos do uso disfuncional dessa substância sobre a 
saúde física -, como também convergente com dados da literatura e com estudo empírico ${ }^{1,33-35}$.

O domínio social do WHOQOL-Bref é constituído apenas por três itens: satisfação do indivíduo com suas relações pessoais (amigos, parentes, colegas, conhecidos), satisfação com a atividade sexual e apoio que recebe de amigos. Os resultados encontrados neste domínio estão de acordo com estudos que apontam declínio da qualidade de vida nos aspectos sociais à medida que aumenta o consumo de alcoólicos ${ }^{7,36-38}$; da mesma forma, corroboram a afirmação da OMS de que o consumo disfuncional de alcoólicos interfere negativamente na qualidade das relações pessoais ${ }^{1}$.

Apesar de a dimensão social do WHOQOL-Bref ter menos itens que os demais domínios - o que, do ponto de vista psicométrico, reduz sua sensibilidade em relação ao WHOQOL-10013,26 -, deve-se ressaltar que somente nesse domínio foram registradas diferenças significantes entre os grupos quando considerado o consumo de alcoólicos no último ano.

\section{Limitações}

A impossibilidade de se mensurar a gravidade da doença hepática no momento da coleta dos dados é uma importante limitação deste estudo, impedindo uma discussão da relação entre condição clínica, senso estrito e qualidade de vida.

Essa impossibilidade ocorreu em função de limitações institucionais no momento da realização do estudo, que restringiram a realização de provas laboratoriais fora do protocolo assistencial do serviço, que indicava essa avaliação apenas para pacientes com maior gravidade clínica. O desenho transversal e a utilização de hepatopatas em tratamento obviamente impedem tanto inferências causais quanto a generalização dos resultados. Dada a extensão do questionário utilizado, optou-se por não avaliar o uso de outras substâncias psicoativas, que pode ser um fator de confundimento das análises efetuadas. Outra limitação a ser considerada foi o pequeno número de sujeitos em certos subgrupos considerados, nomeadamente no subgrupo de maior gravidade alcoólica avaliada pelo AUDIT.

\section{Conclusão}

Os resultados aqui encontrados reforçam achados anteriores de que menor faixa etária, bem como exercício atual de atividade estudantil e/ou empregatícia, se correlaciona a maiores níveis de qualidade de vida, em especial nos domínios físico e social do WHOQOL-Bref. Os mais baixos níveis de qualidade de vida registrados pelos pacientes que informaram consumo de alcoólicos de alto risco ou provável dependência são consistentes com achados da literatura, ainda que neste estudo significância estatística tenha sido evidenciada apenas nos domínios físico (CAGE) e social (AUDIT). A utilização da variável combinada - resultados do CAGE e do AUDIT, ao que se sabe -, pela primeira vez utilizada neste estudo, aponta para a possibilidade de um refinamento da compreensão da relação entre consumo de alcoólicos e qualidade de vida, a ser explorado com desenhos e grupos de sujeitos mais específicos. Assim sendo, os resultados aqui apresentados reforçam a necessidade de pesquisas que controlem a gravidade da doença de base, avalie outros quadros clínicos, psiquiátricos e o uso de outras substâncias psicoativas e o número de pacientes em cada subgrupo considerado.

\section{Referências}

1. World Health Organization. Global strategy to reduce the harmful use of alcohol. Geneva: World Health Organization; 2010.

2. World Health Organization. Global Status Report on Alcohol and Health. Geneva: World Health Organization; 2011.

3. Silveira CM, Silveira CC, Silva JG, Silveira LM, Andrade AG, Andrade LHSG . Epidemiologia do beber pesado e beber pesado episódico no Brasil: uma revisão sistemática da literatura. Rev Psiq Clín. 2008;31(1):31-8.

4. Saatcioglu O, Yapici A, Cakmak D. Quality of life, depression and anxiety in alcohol dependence. Drug Alcohol Rew. 2008;27:83-90.
5. WHOQOL Group. The World Health Organization Quality of Life Assessment (WHOQOL): Development and General Psychometric Properties. Soc Sci Med. 1998;46(12):1569-85.

6. Les I, Doval E, Montserrat F, Jacas C, Cárdenas G, Esteban R, et al. Quality of life in cirrhosis is related to potentially treatable factors. Eur J Gastroenterol Hepatol. 2010;22:221-7.

7. Afendy A, Kallman B, Stepanova M, Younoszai, Z, Aquino RD, Bianchi $\mathrm{G}$, et al. Predictors of health-related quality of life in patients with chronic liver disease. Aliment Pharmacol Ther. 2009;30:469-76.

8. Lam ETP, Lam CLK, Lai CL, Yuen MF, Fong DYT, So TMK. Health-related quality of life of Southern Chinese with chronic hepatitis B infection. Health and quality of life outcomes [Serial da internet] 2009. [Acessado 2011 maio 6]. Disponível em: http//www.hqlo.com/content/7/11/52.

9. David K, Kowdley KV, Unalp A, Kanwai F, Brent EM, Schwinmer JB. Quality of life in adults with nonalcoholic fatty liver disease: baseline data from the NASH CRN. Hepatology. 2009;49(6):1904-12.

10. Eshelman A, Paulson D, Meyer T, Fischer D, Moonka D, Brown K, et al. The influence of alcohol abuse history on the differential, longitudinal patterns of mental and physical quality of life following liver transplantation. Transplant Proc. 2010;42:4145-7.

11. Telles-Correia D, Barbosa A, Meja I, Monteiro E, Barroso E. Mental health and quality of life in alcoholic liver disease patients after liver transplantation: a prospective controlled study. Transplant Proc. 2011;43:184-6.

12. Costenbader EC, Zule WA, Coomes CM. The impact of illicit drug use and harmful drinking on quality of life among injection drug users at high risk for hepatitis C infection. Drug Alcohol Depend. 2007;89:251-8.

13. O'Carroll RE, Smith K, Couston M, Cossar JA, Hayes PC. A comparison of the WHOQOL-100 and the WHOQOL-Bref in detecting change in quality of life following liver transplantation. Qual Life Res. 2000;9:121-4.

14. Taliani G, Rucci P, Biliotti E, Cirrincione L, Aghemo A, Albert A, et al. Therapy expectations and physical comorbidity affect quality of life in chronic hepatitis C virus infection. J Viral Hepat. 2007;14:875-82.

15. Vaillant GE, Hiller-Stermhofel S. The natural history of alcoholism. Alcohol Health Res World. 1996;20(3).

16. Costa JSD, Silveira MF, Gazalle FK, Oliveira SS, Hallal PC, Menezes AMB, et al. Consumo abusivo de álcool e fatores associados: estudo de base populacional. Rev Saude Publica. 2004;38(2):284-91.

17. Laranjeira R, Pinsky I, Sanches M, Zaleski RC. Padrão do uso de álcool em brasileiros adultos. Rev Bras Psiquiatr. 2010;32(3):231-45.

18. Marin-León L, Oliveira HB, Botega NJ. Mortalidade por dependência de álcool no Brasil: 1998-2002. Psicol Estud. 2007;12(1):115-21.

19. Ewing JA. Detecting alcoholism, the CAGE questionnaire. JAMA. 1984;252:1905-7.

20. Ribeiro MS. O uso funcional e disfuncional de substâncias psicoativas. In: Ribeiro MS. Ferramentas para descomplicar a atenção básica em saúde mental. Juiz de Fora: Editora da UFJF; 2007.

21. Mayfield DG, MacLeod G, Hall P. The CAGE questionnaire: validation of a new alcoholism screening instrument. Am J Psychiatry. 1974;131:1121-3.

22. Masur J, Monteiro MG. Validation of the "CAGE" alcoholism screening test in a Brazilian psychiatric inpatient hospital setting. Braz J Med Biol Res. 1983;16(3):189-282.

23. Mendez EB. Uma versão brasileira do AUDIT - Alcohol disorders identification test [dissertação]. Rio Grande do Sul: Universidade Federal de Pelotas; 1999.

24. Saunders JB, Aasland OG. World Health Organization Collaborative project on the identification and treatment of persons with harmful alcohol consumption - Report on phase I. Development of a screening instrument. Geneva: World Health Organization; 1987.

25. World Health Organization. WHOQOL - User Manual. Division of Mental Health and prevention of substance abuse. Geneva: World Health Organization; 1998.

26. Fleck, MPA, Louzada S, Xavier M, Chachamovich E, Vieira G, Santos $\mathrm{L}$, et al. Aplicação da versão em português do instrumento abreviado de avaliação da qualidade de vida "WHOQOL-Bref". Rev Saude Publica. 2000;34(2):178-83.

27. Conover WJ. Practical nonparametric statistics. 3rd ed. New York: Wiley; 1999.

28. Cruz LN, Polanczyk CA, Camey AS, Hoffman JF, Fleck MP. Quality of life in Brazil normative values for the WHOQOL-Bref in a southern general population sample. Qual Life Res. [Serial da internet] 2011. 
[Acessado 2011 jun 6]. Disponível em: http:www.springerlink.com/ content/r73p45wt3h242862/.

29. Rocha NS, Fleck MPA. Avaliação de qualidade de vida e importância dada a espiritualidade/religiosidade/crenças pessoais (SRPB) em adultos com e sem problemas crônicos de saúde. Rev Psiq Clín. 2011;38(1):19-23.

30. Sung $\mathrm{H}$, Chang $\mathrm{M}$, Saab S. Management of hepatitis $\mathrm{C}$ antiviral therapy adverse effects. Curr Hepat Rep. 2011;10:33-40.

31. Kollia Z, Patelarou E, Vivilaki V, Kollia E, Kefou F, Elefsiniotis F, et al. Translation and validation of the Greek chronic liver disease questionnaire. World J Gastroenterol. 2010;16(46):5838-44.

32. Ohaeri JU, Awadalla AW, Gado OM. Subjective quality of life in a nationwide sample of Kuwaiti subjects using the short version of the WHO quality of life instrument. Soc Psychiatry Psychiatr Epidemiol. 2009;44:693-701.

33. Akvardar Y, Akdede BB, Ozerdem A, Eser E, Topkaya S, Alptekin K. Assessment of quality of life with the WHOQOL-Bref in a group of
Turkish psychiatric patients compared with diabetic and healthy subjects. Psychiatry Clin Neurosci. 2006;60:693-9.

34. World Health Organization. Who Global Status Report on Alcohol. Department of Mental Health and Substance Abuse. Geneva: World Health Organization; 2004.

35. Room R, Babor T, Rehm J. Alcohol and public health. Lancet. 2005;365:519-30.

36. Foster JH, Powell JE, Marshall EJ, Peters TJ. Quality of life in alcohol: dependent subjects: a review. Qual Life Res. 1999;8(3):255-61.

37. Silva Lima AFB, Fleck MPA, Pechansky F, De Boni R, Sukop P. Psychometric Properties of the World Health Organization Quality of Life instrument (WHOQOL-Bref) in alcoholic males: a pilot study. Qual Life Res. 2005;14:473-8.

38. Fontes A, Figlie NB, Laranjeira R. O comportamento de beber entre dependentes de álcool: estudo de seguimento. Rev Psiq Clín. 2006;33(6):304-12. 